\title{
Two-dimensional speckle tracking echocardiography derived post systolic shortening in patients with unstable angina and normal left ventricular systolic function
}

\author{
Srinivasan Giridharan (D), Selvaraj Karthikeyan (D), Arumugam Aashish (D), \\ Balasubramaniyan Amirtha Ganesh (D, Palamalai Arun Prasath (D), Pandiyan Usha (D) \\ Department of Cardiology, Mahatma Gandhi Medical College and Research Institute; Puducherry-India
}

ABSTRACT

Objective: Post-systolic shortening (PSS) has been shown to be sensitive in detecting ischemia on stress echocardiography. In this study, we aimed to assess the diagnostic potential of resting PSS and post-systolic index (PSI) in patients with suspected unstable angina (UA).

Methods: A total of 159 participants with suspected UA without any wall motion abnormalities were recruited. They all underwent speckle tracking echocardiography (STE) and coronary angiogram (CAG). Global longitudinal strain (GLS); presence or absence of pathological PSS; PSI ${ }_{17}$ and PSI ${ }_{12}$ and PSI in left anterior descending, left circumflex, and right coronary artery territories were assessed. On the basis of CAG, those who had more than $70 \%$ stenosis were labelled to have obstructive CAD.

Results: Obstructive disease was noted in $54.7 \%$ patients. The prevalence of PSS (62.1\% vs. $13.9 \%$ ), mean PSI $_{17}(5.4$ vs. 3.3$)$, and PSI 12 (6.2 vs. 3.7$)$ were significantly higher in those with CAD compared with patients without obstructive disease. Both PSS [odds ratio (OR) 10.145; 95\% confidence interval (CI) 4.577-22.489; $\mathrm{p}=0.001$ ] and $\mathrm{PSI}_{17}$ (OR: 1.217; 95\% Cl: 1.064-1.393; $\mathrm{p}=0.004$ ) were predictors of CAD by multivariate regression analysis. PSS had a sensitivity of $62.1 \%$ and specificity of $86.1 \%$ with a positive predictive value of $84.4 \%$. PSI $_{17}$ [area under curve (AUC): $0.637 ; p=0.003$ ] and $\mathrm{PSI}_{12}$ (AUC: $0.661 ; \mathrm{p}=0.001$ ) have moderate accuracy in identifying obstructive CAD.

Conclusion: In patients presenting with suspected UA, STE derived PSS has reasonable sensitivity and good specificity in diagnosis of obstructive CAD. Patients identified to have PSS can be subjected to CAG without further stress testing because of its high positive predictive value.

Keywords: post-systolic shortening, speckle tracking echocardiography, unstable angina

Cite this article as: Giridharan S, Karthikeyan S, Aashish A, Ganesh BA, Prasath PA, Usha P. Two-dimensional speckle tracking echocardiography derived post systolic shortening in patients with unstable angina and normal left ventricular systolic function. Anatol J Cardiol 2021; 25: 880-6.

\section{Introduction}

Coronary artery disease (CAD) is the most common cause of mortality both worldwide and in India (1). Unstable angina (UA) constitutes an important sub-group among patients with CAD presenting with chest pain to both the emergency room (ER) and outpatient department. In patients with definite acute coronary syn- drome (ACS), resting ECG may be non-diagnostic in more than half of the patients. Hence, several non-invasive investigations are used in the diagnosis and risk stratification of suspected UA without any high-risk features before subjecting them to an invasive investigation, such as coronary angiogram (CAG), although patients with high-risk features such as ongoing pain, dynamic ECG changes, and regional wall motion abnormalities (RWMA) undergo CAG 


\section{HIGHLIGHTS}

- $\quad$ Stress induced post-systolic shortening (PSS) is known to be sensitive in detecting myocardial ischemia.

- In this study, we aimed to assess the diagnostic potential of speckle tracking echocardiography derived resting PSS in patients with unstable angina.

- PSS has a reasonable sensitivity and good specificity in identifying obstructive coronary artery disease.

without any non-invasive testing (2). Except for stress ECG which has limited sensitivity and specificity, the use of other imaging modalities (single photon emission computed tomography/computed tomography/magnetic resonance imaging) is restricted in developing countries like ours owing to financial constraints, availability, and lack of technical expertise. This potential gap in resources has always propelled clinicians to search for a point of care and cost effective tool to diagnose CAD.

Impairment in several 2-dimensional and 3-dimensional strain parameters have been shown to have a role in predicting obstructive CAD, both in stable ischemic heart disease (3) and non-ST elevation myocardial infarction (NSTEMI)/ST elevation myocardial infarction (STEMI) patients (4). Kukulski et al. (5) first described the diagnostic superiority of tissue Doppler derived post-systolic index (PSI) over conventional strain parameters in identifying ischemia during coronary angioplasty. Speckle tracking echocardiography (STE) derived post-systolic shortening (PSS) was reported to be a promising marker in detecting myocardial ischemia (6). Myocardial shortening that happens after aortic valve closure is defined as PSS. It is easily identifiable by STE and observed during isovolumic relaxation. PSS can be measured and quantified objectively by PSI. Not only is PSS a marker for detecting myocardial ischemia, but also elevated PSI values in segments with RWMA represent actively contracting myocardium and hence correlates well with viability (7). However, the use of PSS has not been described in patients with UA with normal left ventricle (LV) function presenting to the ER. In this study, our objective was to document the role of STE derived PSS and PSI at rest in the diagnosis of CAD among patients with suspected UA and in their risk stratification.

\section{Methods}

\section{Study population}

This was a prospective study with 159 patients, older than 18 years of age with suspected unstable angina and normal LV systolic function who were admitted for a coronary angiogram. All those included had no RWMA on echocardiogram and negative highly sensitive troponin assay result.

Patients with a previous history of myocardial infarction, known $C A D$, presence of significant valvular heart disease, patients on chemotherapy, those with stage 4 and 5 chronic kidney disease, presence of left bundle branch block/pacemaker, and atrial fibrillation were excluded. The study was approved by the Institutional Ethics Committee, Mahatma Gandhi Medical College and Research Institute (Date: 21.05.2020). All the participants signed an informed consent form.

Baseline parameters like the patients' age, sex, body mass index (BMI), presence of risk factors such as diabetes mellitus, systemic hypertension, dyslipidaemia, obesity, current smoking status, and other relevant clinical data were collected.

\section{Speckle tracking echocardiogram}

All the study participants underwent echocardiogram in a Vivid E-95 system (GE healthcare) equipped with a 4V phased array matrix transducer (1.5-4.0 MHz) just before the coronary angiogram. Echocardiographic measurements were obtained according to American Society of Echocardiography guidelines (8). The images were stored and later analysed by a single cardiologist experienced in strain echocardiogram, who was blinded to the patient's clinical and angiographic details. Cine loops with 3 cardiac cycles in apical 3 chamber, apical 4 chamber, and apical 2 chamber views were recorded at a frame rate of 50-80 fps and stored. In each of the apical planes, tracing of LV myocardium was automatically performed by the software. Manual adjustments were made by the investigator whenever tracking the region of interest was inadequate. The longitudinal strain curves were generated, and the presence of PSS was categorised on the basis of the criteria laid out by Voigt et al. (9). The PSI was derived from the strain curve as [(peak negative strain in cardiac cycle - peak negative strain in systole)]/(peak negative strain in cardiac cycle) $\times 100$ (see Fig. 1). A 17 segment bulls' eye map with PSI of individual segments was generated automatically by the software, and the mean PSI was calculated as an average of the individual segments. The patients were determined to have PSS if PSI in any one of the 17 segments was more than $20 \%$. $\mathrm{PSI}_{12}$ was derived manually from 6 basal and 6 mid-ventricular segments. On the basis of the standardised model of myocardial perfusion territories (10), region-specific PSI was calculated as the average value of the segments belonging to each perfusion territory in the left anterior descending artery (LAD), left circumflex artery (LCX), and right coronary artery (RCA), respectively. For example, the region specific PSI of RCA was calculated manually by averaging the PSI values of the 5 segments perfused by the RCA (basal inferior and inferoseptal, mid inferior and inferoseptal, and apicoinferior). Similarly, the region specific PSI of LCX territory was calculated from the following 5 segments - basal and mid inferolateral, basal and mid anterolateral, and apicolateral.

\section{Coronary angiogram}

The decision to catheterize a subject with UA was made by the admitting clinician. The research group had no role in deciding the need for invasive strategy. Only after the patient was admitted for CAG were they included in the study and after signing the informed consent. The decision to perform CAG was based on the clinical 


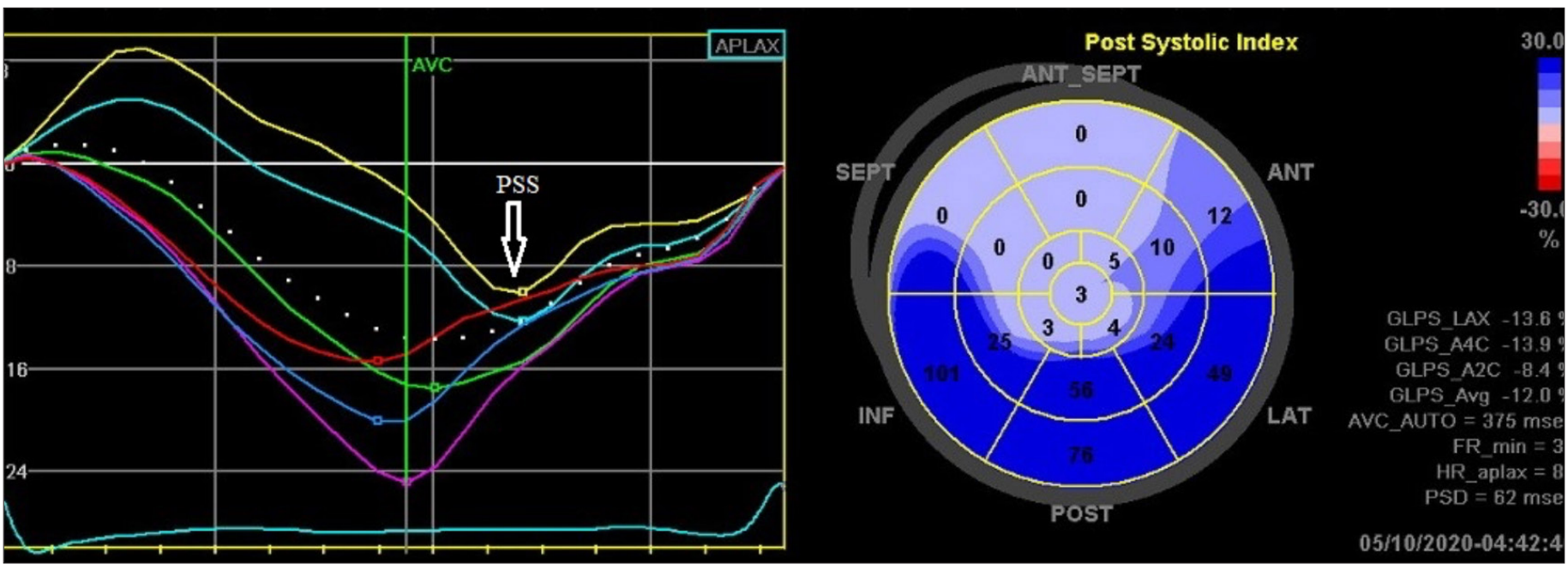

Figure 1. Two-dimensional speckle tracking echocardiography revealing post-systolic shortening. Right panel displays the bull's eye map of segmental post systolic index

presentation (typical angina), risk factors, and ECG changes and was not based on the STE results. Angiographic images were studiously assessed by an interventionist who was blinded to the patient data. The study participants were divided into 2 groups on the basis of CAG results. The patients were diagnosed to have obstructive CAD if they had a lesion of $>70 \%$ stenosis in one or more major epicardial coronary vessels or $50 \%-70 \%$ stenosis but with less than TIMI 3 flow. Participants with normal coronaries and non-obstructive disease were taken as the control group. Patients with $>70 \%$ stenosis in obtuse marginal, posterior descending artery, posterolateral branch, and ramus were considered obstructive only if the vessel diameter was $>2.25 \mathrm{~mm}$. SYNTAX I score was calculated using the standard online calculator from the website http://www.syntaxscore.com/calculator/start.htm.

\section{Statistical analysis}

The data analysis was performed using Statistical Package for Social Sciences for Windows, version 16.0 (SPSS Inc., Chicago, USA). Quantitative variables were expressed as mean \pm standard deviation, whereas categorical variables were expressed as numbers and percentages. Continuous variables were compared using independent samples t-test. Pearson chi-squared test and Fisher exact tests were used for categorical variables. A value of $p<0.05$ was considered to indicate a statistically significant difference. Binary logistic regression analysis was done to study the predictors of obstructive CAD. Linear correlation between PSI and SYNTAX score was measured by Spearman S rank correlation coefficient. Receiver operator characteristic curves (ROCs) were constructed for strain parameters to detect their sensitivity and specificity in identifying obstructive CAD.

\section{Results}

A total of 159 patients with suspected UA were included for final analysis after excluding 16 patients owing to issues with image quality. The clinical details stratified by presence or absence of PSS are provided in Table 1. The mean age was 55.9 years, and only $22.7 \%$ were women. The GRACE score of the included participants ranged from 35 to 126 suggesting that the study participants predominantly had low-risk unstable angina. Thirty-five patients $(21.8 \%)$ had ischemic changes on ECG, whereas the rest had normal or non-diagnostic changes. Only $17.6 \%(\mathrm{n}=28)$ had non-invasive ischemia testing with another 12 patients (7.5\%) undergoing CT-CAG before conventional CAG.

Of the 159 patients studied, $54.7 \%(n=87)$ had obstructive disease and $45.3 \%$ ( $n=72$ ) had normal/non-obstructive disease. Single vessel involvement was noted in $43.7 \%(n=38)$. Double vessel and triple vessel disease were noted in $31 \%(n=27)$ and $20.7 \%(n=18)$, respectively. Only $4.6 \%(n=4)$ of those with obstructive CAD had left main disease. Of the 87 with obstructive disease, 9 patients were put on medical management, and the rest were advised to undergo revascularisation (22 for CABG and 56 for PCI).

Among the risk factors, patients with PSS more often had diabetes and hypertension, and they were more frequently on beta-blockers and statin therapy (see Table 1). Furthermore, higher systolic blood pressure, E/e', and significantly altered global longitudinal strain (GLS) (less negative) were noted in those with PSS. The mean LV ejection fraction was $64.2 \%$ suggesting that the study group had only patients with normal LV systolic function, and there was no significant difference between those with or without PSS. Those with PSS had higher occurrence of obstructive disease overall and also had significant lesions in LAD, LCX, and RCA than those without PSS. The prevalence of PSS and the mean $\mathrm{PSI}_{17}$ and $\mathrm{PSI}_{12}$ were all significantly high in patients with obstructive lesions (Table 2).

Both $\mathrm{PSI}_{17}$ and $\mathrm{PSI}_{12}$ were predictors of obstructive CAD (Table 3) by regression analysis after adjusting for baseline clinical and echocardiographic variables. Categorical PSS was the strongest predictor of $\mathrm{CAD}(\mathrm{OR}=10.1 ; 95 \% \mathrm{Cl}=4.58-22.49$; 


\begin{tabular}{|c|c|c|c|c|}
\hline & Presence of PSS ( $n=64)$ & Absence of PSS ( $n=95)$ & Total patients ( $n=159)$ & $P$-value \\
\hline Age, years & $57.2 \pm 9.1$ & $54.7 \pm 10.0$ & $55.9 \pm 8.9$ & 0.116 \\
\hline Body mass index, $\mathrm{kg} / \mathrm{m}^{2}$ & $25.3 \pm 3.1$ & $24.4 \pm 2.5$ & $24.8 \pm 2.8$ & 0.085 \\
\hline Diabetes mellitus & $41(64.1 \%)$ & $46(48.4 \%)$ & $87(54.7 \%)$ & 0.052 \\
\hline Dyslipidemia & $15(23.4 \%)$ & $23(24.2 \%)$ & $38(23.9 \%)$ & 0.911 \\
\hline Smoking & $7(10.9 \%)$ & $14(14.7 \%)$ & $21(13.2 \%)$ & 0.488 \\
\hline Systolic blood pressure & $138.16 \pm 20.8$ & $131.24 \pm 17.9$ & $134 \pm 19.3$ & 0.031 \\
\hline Diastolic blood pressure & $84.5 \pm 8.9$ & $81.8 \pm 10.4$ & $83 \pm 9.9$ & 0.105 \\
\hline Beta-blocker & $39(60.9 \%)$ & $43(45.3 \%)$ & $82(51.6 \%)$ & 0.052 \\
\hline \multicolumn{5}{|l|}{ Echocardiogram } \\
\hline $\mathrm{EF}$ & $64.4 \% \pm 3.5$ & $64.1 \% \pm 4.5$ & $64.25 \pm 4.1$ & 0.601 \\
\hline $\mathrm{E} / \mathrm{e}^{\prime}$ & $12.0 \pm 4.6$ & $10.3 \pm 3.0$ & $11.0 \pm 3.8$ & 0.015 \\
\hline LV mass index & $102.7 \pm 24.6$ & $97.0 \pm 23.3$ & $99.3 \pm 23.9$ & 0.145 \\
\hline GLS & $-14.7 \% \pm 10.7$ & $-18.5 \% \pm 8.6$ & $-16.9 \% \pm 9.7$ & 0.021 \\
\hline \multicolumn{5}{|l|}{ CAG } \\
\hline Obstructive CAD & $54(84.4 \%)$ & $33(34.7 \%)$ & $87(54.7 \%)$ & 0.001 \\
\hline
\end{tabular}

\begin{tabular}{|c|c|c|c|c|}
\hline & $\begin{array}{l}\text { Obstructive } \\
\text { CAD }(n=87)\end{array}$ & $\begin{array}{c}\text { Non-obstructive } \\
\text { CAD }(n=72)\end{array}$ & $\begin{array}{c}\text { Total } \\
(\mathrm{n}=159)\end{array}$ & $P$-value \\
\hline $\begin{array}{l}\text { Categorical } \\
\text { PSS }\end{array}$ & $54(62.1 \%)$ & $10(13.9 \%)$ & $64(40.3 \%)$ & 0.001 \\
\hline $\mathrm{PSI}_{17}$ & $5.4 \pm 5.5$ & $3.3 \pm 2.5$ & $4.5 \pm 4.5$ & 0.002 \\
\hline $\mathrm{PSI}_{12}$ & $6.2 \pm 6.1$ & $3.7 \pm 2.9$ & $5.1 \pm 5.0$ & 0.001 \\
\hline PSI LAD & $5.6 \pm 6.9$ & $3.2 \pm 2.9$ & $4.5 \pm 5.6$ & 0.003 \\
\hline PSI LCX & $7.1 \pm 9.2$ & $4.2 \pm 5.1$ & $5.8 \pm 7.8$ & 0.012 \\
\hline PSI RCA & $3.5 \pm 4.9$ & $2.6 \pm 3.9$ & $3.1 \pm 4.5$ & 0.105 \\
\hline
\end{tabular}

$\mathrm{p}=0.001$ ). Presence of PSS had a reasonable sensitivity with good specificity and positive predictive value (Table 4).

Both $\mathrm{PSI}_{17}$ and $\mathrm{PSI}_{12}$ had modest accuracy in diagnosing obstructive CAD with an area under the curve (AUC) of 0.637 (95\%
Table 3. Logistic regression analysis for predicting obstructive CAD

\begin{tabular}{|lcc|}
\hline & Adjusted $\mathbf{O R}^{*}$ (95\% $\mathbf{~ C l )}$ & $\boldsymbol{P}$-value \\
\hline PSI $_{17}$ & $1.217(1.064-1.393)$ & 0.004 \\
PSI $_{12}$ & $1.191(1.049-1.353)$ & 0.007 \\
PSI LAD & $1.171(1.052-1.304)$ & 0.004 \\
PSI LCX & $1.069(1.000-1.143)$ & 0.049 \\
PSI RCA & $1.067(0.973-1.171)$ & 0.169 \\
\hline *Adjusted for age, sex, blood pressure, presence of hypertension, diabetes, and \\
echocardiographic parameters (E/e', left ventricle mass index, and global longitudinal strain). \\
OR - odds ratio; Cl - confidence interval; CAD - coronary artery disease \\
\hline
\end{tabular}

Cl: $0.551-0.723 ; p=0.003$ ) and 0.661 (95\% Cl: $0.576-0.745 ; \mathrm{p}=0.001$ ), respectively (Fig. $2 \mathrm{a}$ and $2 \mathrm{~b}$ ). A mean $\mathrm{PSI}_{17}$ of more than 3.1 had a sensitivity and specificity of $61 \%$ and $53.5 \%$, respectively, whereas mean $\mathrm{PSI}_{12}>3.1$ had a sensitivity and specificity of $70.1 \%$ and $50.7 \%$, respectively. The accuracy of territorial PSI (PSI LAD) in identifying significant lesions of LAD (Fig. 2C) was poor with an AUC of 0.575 (95\% Cl: $0.484-0.665 ; p=0.112$ ). Similarly, both PSI LCX 


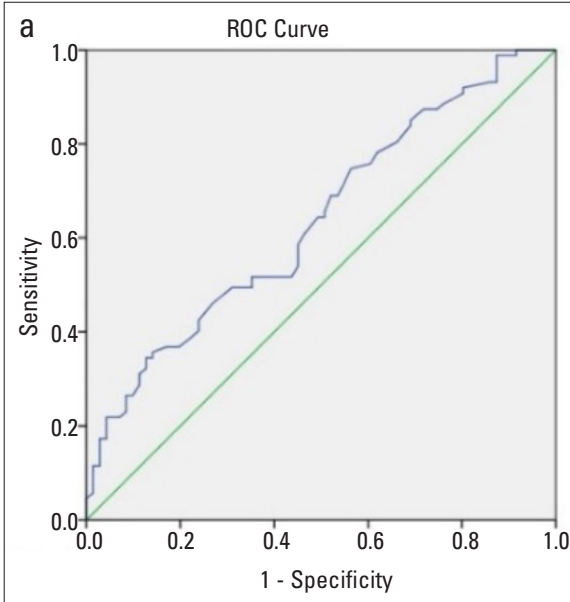

Diagonal segments are produced by ties

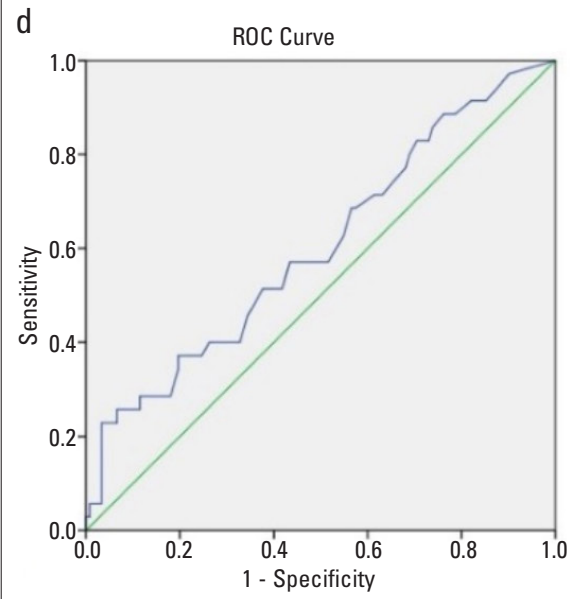

Diagonal segments are produced by ties

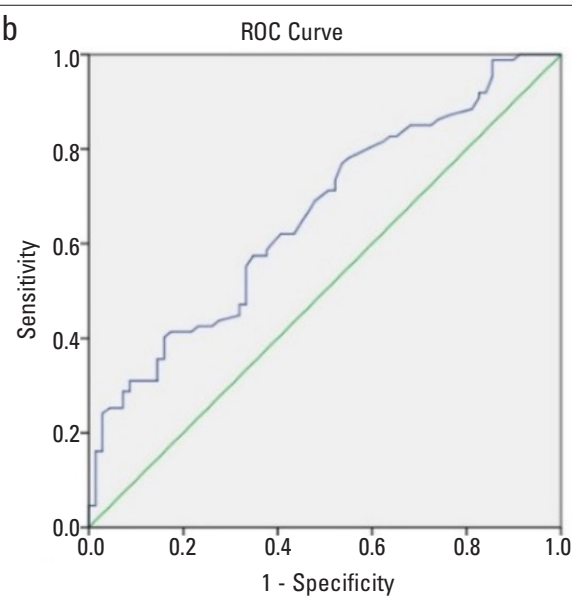

Diagonal segments are produced by ties

e

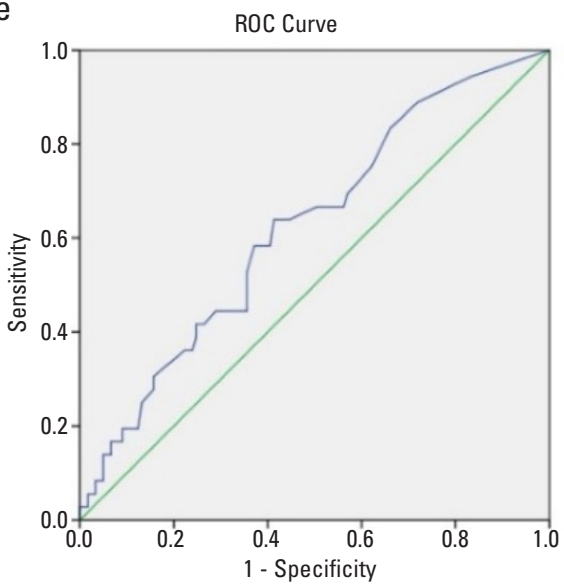

Diagonal segments are produced by ties

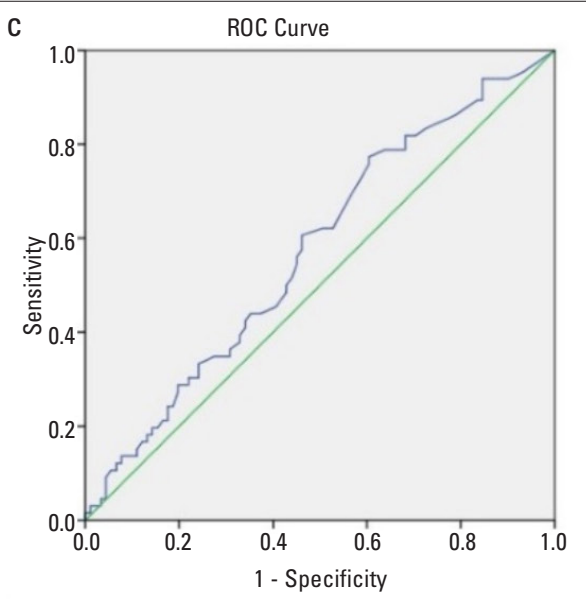

Diagonal segments are produced by ties

Figure 2. Receiver operating characteristic curve for the diagnosis of coronary artery disease using PSI ${ }_{17}$ and PSI 12 (2a and $\left.2 b\right)$; PSI LAD, PSI LCX, PSI RCA in identification of obstructive disease in their corresponding territories $(2 c, 2 d, 2 e$ respectively)

PSI - post-systolic index; LAD - left anterior descending artery; LCX - left circumflex artery; RCA - right coronary artery

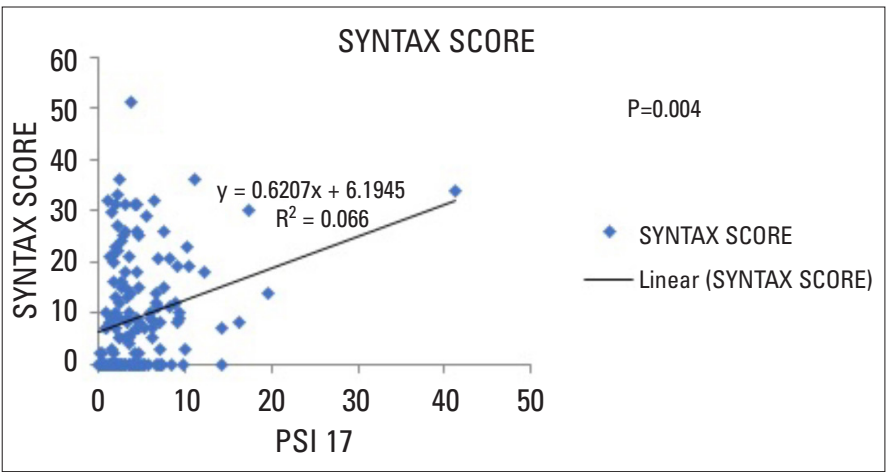

Figure 3. Correlation between post-systolic index (PSI) ${ }_{17}$ and SYNTAX score

Table 4. Diagnostic accuracy of PSS in identifying obstructive CAD

\begin{tabular}{|lccccc|}
\hline & Sensitivity & Specificity & PPV & NPV & Accuracy \\
\hline $\begin{array}{l}\text { Presence } \\
\text { of PSS }\end{array}$ & $62.1 \%$ & $86.1 \%$ & $84.4 \%$ & $65.3 \%$ & $73.0 \%$ \\
\hline
\end{tabular}

PSS - post-systolic shortening; CAD - coronary artery disease; PPV - positive predictive value; NPV - negative predictive value and PSI RCA had only moderate accuracy in pointing out obstructive diseases of respective coronary territory with AUC of 0.602 (95\% Cl: $0.493-0.711 ; p=0.065$ ) and 0.624 (95\% Cl: 0.522-0.725; $\mathrm{p}=0.024$ ), respectively (Fig. $2 \mathrm{~d}$ and $2 \mathrm{e}$ ).

There was no significant difference in both mean $\mathrm{PSI}_{17}$ and $\mathrm{PSI}_{12}$ values between those with single and multivessel disease (PSI $_{17}-5.4 \pm 6.5$ vs. $5.4 \pm 3.8, p=0.873$; $\mathrm{PSI}_{12}-6.1 \pm 6.9$ vs. $6.5 \pm 4.9$, $\mathrm{p}=0.751$ ). As shown in Figure 3 , the correlation between SYNTAX score and mean $\mathrm{PSI}_{17}$ was weak though being significant statistically $\left(R^{2}=0.066, p=0.004\right)$.

\section{Discussion}

The salient data generated from this study were PSS as depicted by high PSI values was significantly more frequent in patients with obstructive CAD; mean $\mathrm{PSI}_{17}$ and $\mathrm{PSI}_{12}$ along with territorial PSI of LAD and LCX were statistically higher in those with CAD; multivariate regression analysis suggested high PSI values indicating the presence of PSS to be a strong predictor 
of obstructive disease; although the accuracy of PSS to diagnose CAD is moderate, it has high specificity and positive predictive value. To the best of our knowledge, this is the first study providing information on the diagnostic role of STE derived PSS at rest in patients with UA.

Prior animal studies have demonstrated the sensitivity of postsystolic deformation in acutely ischemic myocardium (11). Postsystolic thickening have been documented in patients with critically stenosed arteries and identified as a sign of myocardial viability (12). A large database exists regarding the role of dobutamine stress echocardiography (DSE) induced PSS in patients with CAD $(12,13)$. Strain rate imaging during DSE has been investigated, and the ratio of PSS to maximal segmental deformation was found to be the best quantitative parameter in diagnosing stress induced ischemia (14). In another pivotal study using pulse wave Doppler imaging derived PSS, stress-induced PSS was the most sensitive index in the diagnosis of induced ischemia (13). However, there is a paucity of data about the accuracy of post-systolic deformation indices derived with resting STE in patients suspected to have UA.

PSS, albeit a sensitive marker of ischemia, is not $100 \%$ specific for it. In a seminal study by Voigt et al. (9), almost one-third patients $(31 \%)$ in the control group had PSS which was considered to be physiologic. This non-specificity hinders the widespread use of PSS in clinical practice. Hence with an intention to exclude segments showing physiologic PSS, strict criteria of PSI exceeding $20 \%$ of the total strain during cardiac cycle as suggested by Voigt et al. (9) was employed in our study. Few other studies have used this threshold to label PSS and reported its role in both stable angina and ST elevation myocardial infarction (15-17). Applying these criteria led to a PSS of $40.3 \%$ in our study population (62.1\% in CAD group vs $13.9 \%$ in control group). The prevalence of CAD was low in our cohort (54.7\%) owing to the strict inclusion criteria of only those with suspected UA and normal LV function with no RWMA.

The higher sensitivity of PSS reported in literature are predominantly from stress induced strain imaging studies. In a study by Onishi et al. (18), the sensitivity to detect CAD improved from $62 \%$ at rest to $89 \%$ at peak by dobutamine stress echocardiography with a specificity of $77 \%$ and $73 \%$, respectively. In terms of information on STE derived PSS at rest, there is only one other study which has reported the sensitivity and specificity of $\mathrm{PSS}_{18}$ as $65 \%$ and $67 \%$, respectively; however, it was derived from a group of patients with suspected stable CAD (17). In spite of PSS being a strong predictor of CAD, it has only modest sensitivity $(62.1 \%)$ as reported by Brainin et al. (17) previously. However, its specificity is quite high ( $86.1 \%$ ) using the criteria of Voigt et al. (9) to identify pathologic PSS. This has immense clinical significance as patients suspected to have UA and identified to have PSS can be advised to undergo CAG without any further non-invasive testing.

Similarly, mean PSI derived from both 17 segments as well as 12 segments had only modest accuracy in diagnosing obstructive CAD; however, a high cut-off value of $\mathrm{PSI}_{17}$, for example, $>4.75$ and
$\mathrm{PSI}_{12}>5.45$ in our study had a good specificity of $80.3 \%$ and $78.3 \%$, respectively, which has relevance from a clinician's perspective.

The accuracy of regional PSI of LAD, LCX, and RCA in the diagnosis of CAD was reported to be excellent with AUC varying between 0.81 to 0.99 (based on the segment chosen) in DSE study (13). There are no available data apart from ours on the role of regional PSI at rest. From our study, regional PSI (LAD, LCX, RCA) has only modest accuracy in localization of the involved vessels. This could be because of a larger percentage of patients having multi-vessel disease $(56.3 \%)$, or there could always be an overlap in the segments vascularised by these arteries between individual patients based on dominance.

The main strength of this study apart from its prospective nature and large sample size is that it precisely included those patients with suspected UA (negative troponin), normal LV function (no RWMA), with normal/non-diagnostic resting ECG (almost $80 \%$ ). There is a dilemma regarding the need for cardiac catheterisation in these patients in a developing country like ours owing to non-availability of non-invasive imaging modalities (such as SPECT, PET, MRI, and CT). All our data were acquired at rest, which lends more clinical relevance as it can be easily performed bedside in an ER setting.

\section{Study limitations}

The major limitation of our study is that we did not aim to compare post systolic deformation indices with other standard strain parameters. Second, the significance of the lesion was solely on the basis of angiographic criteria and not functionally substantiated (no fractional flow reserve done). Future studies should look into association between functionally significant lesion and PSS. Third, we did not follow up with any of these patients for cardiac events.

\section{Conclusion}

In patients with low-risk UA and no RWMA, resting PSS by STE has reasonable sensitivity and good specificity in the diagnosis of obstructive CAD. Similarly, higher cut-off values of mean $\mathrm{PSI}_{17}$ and $\mathrm{PSI}_{12}$ as suggested above has good specificity. Hence, those patients with altered pathologic post-systolic indices can be considered for CAG without submitting them for non-invasive imaging.

Acknowledgments: Dr. Arun Sugumaran for assisting us in Statistical analysis for this study.

Conflict of interest: None declared.

Peer-review: Externally peer-reviewed.

Author contributions: Concept - S.G., B.A.G., P.A.P.; Design - S.G., A.A., P.A.P.; Supervision - S.G., S.K., A.A., B.A.G., P.U.; Fundings - None; Materials - P.U.; Data collection \&/or processing - S.K., P.U.; Analysis \&/ or interpretation - S.G., P.A.P.; Literature search - S.G., A.A.; Writing S.G., A.A., P.A.P.; Critical review - S.K., B.A.G. 


\section{References}

1. Lozano R, Naghavi M, Foreman K, Lim S, Shibuya K, Aboyans V, et al. Global and regional mortality from 235 causes of death for 20 age groups in 1990 and 2010: a systematic analysis for the Global Burden of Disease Study 2010. Lancet 2012; 380: 2095-128. [Crossref]

2. Collet JP, Thiele H, Barbato $E$, Barthélémy 0 , Bauersachs J, Bhatt DL, et al.; ESC Scientific Document Group. 2020 ESC Guidelines for the management of acute coronary syndromes in patients presenting without persistent ST-segment elevation. Eur Heart J 2021; 42: 1289-367. [Crossref]

3. Moustafa S, Elrabat K, Swailem F, Galal A. The correlation between speckle tracking echocardiography and coronary artery disease in patients with suspected stable angina pectoris. Indian Heart $\mathrm{J}$ 2018; 70: 379-86. [Crossref]

4. Caspar T, Samet H, Ohana M, Germain P, El Ghannudi S, Talha S, et al. Longitudinal 2D strain can help diagnose coronary artery disease in patients with suspected non-ST-elevation acute coronary syndrome but apparent normal global and segmental systolic function. Int J Cardiol 2017; 236: 91-4. [Crossref]

5. Kukulski T, Jamal F, Herbots L, D'hooge J, Bijnens B, Hatle L, et al. Identification of acutely ischemic myocardium using ultrasonic strain measurements. A clinical study in patients undergoing coronary angioplasty. J Am Coll Cardiol 2003; 41: 810-9. [Crossref]

6. Okuda K, Asanuma T, Hirano T, Masuda K, Otani K, Ishikura F, et al. Impact of the coronary flow reduction at rest on myocardial perfusion and functional indices derived from myocardial contrast and strain echocardiography. J Am Soc Echocardiogr 2006; 19: 781-7. [Crossref]

7. Skulstad H, Edvardsen T, Urheim S, Rabben SI, Stugaard M, Lyseggen $\mathrm{E}$, et al. Postsystolic shortening in ischemic myocardium: active contraction or passive recoil? Circulation 2002; 106: 718-24. [Crossref]

8. Voigt JU, Pedrizzetti G, Lysyansky P, Marwick TH, Houle H, Baumann $\mathrm{R}$, et al. Definitions for a common standard for 2D speckle tracking echocardiography: consensus document of the EACVI/ASE/ Industry Task Force to standardize deformation imaging. J Am Soc Echocardiogr 2015; 28: 183-93. [Crossref]

9. Voigt JU, Lindenmeier G, Exner B, Regenfus M, Werner D, Reulbach $U$, et al. Incidence and characteristics of segmental postsystolic longitudinal shortening in normal, acutely ischemic, and scarred myocardium. J Am Soc Echocardiogr 2003; 16: 415-23. [Crossref]
10. Cerqueira MD, Weissman NJ, Dilsizian V, Jacobs AK, Kaul S, Laskey WK, et al.; American Heart Association Writing Group on Myocardial Segmentation and Registration for Cardiac Imaging. Standardized myocardial segmentation and nomenclature for tomographic imaging of the heart. A statement for healthcare professionals from the Cardiac Imaging Committee of the Council on Clinical Cardiology of the American Heart Association. Circulation 2002; 105: 539-42. [Crossref]

11. Akaishi M, Weintraub WS, Schneider RM, Klein LW, Agarwal JB, Helfant $\mathrm{RH}$. Analysis of systolic bulging. Mechanical characteristics of acutely ischemic myocardium in the conscious dog. Circ Res 1986; 58: 209-17. [Crossref]

12. Barletta G, Del Bene R, Lo Sapio P, Gallini C, Fantini F. Post-ejection thickening as a marker of viable myocardium. An echocardiographic study in patients with chronic coronary artery disease. Basic Res Cardiol 1998; 93: 313-24. [Crossref]

13. Celutkiene J, Sutherland GR, Laucevicius A, Zakarkaite D, Rudys A, Grabauskiene V. Is post-systolic motion the optimal ultrasound parameter to detect induced ischaemia during dobutamine stress echocardiography? Eur Heart J 2004; 25: 932-42. [Crossref]

14. Voigt JU, Exner B, Schmiedehausen K, Huchzermeyer C, Reulbach $\mathrm{U}$, Nixdorff U, et al. Strain-rate imaging during dobutamine stress echocardiography provides objective evidence of inducible ischemia. Circulation 2003; 107: 2120-6. [Crossref]

15. Brainin P, Biering-Sørensen SR, Møgelvang $R$, Søgaard $P$, Jensen JS, Biering-Sørensen T. Postsystolic Shortening by Speckle Tracking Echocardiography Is an Independent Predictor of Cardiovascular Events and Mortality in the General Population. J Am Heart Assoc 2018; 7: e008367. [Crossref]

16. Brainin $P$, Haahr-Pedersen $S$, Sengeløv $M$, Olsen FJ, Fritz-Hansen $T$ Jensen JS, et al. Presence of post-systolic shortening is an independent predictor of heart failure in patients following ST-segment elevation myocardial infarction. Int J Cardiovasc Imaging 2018; 34: 751-60. [Crossref]

17. Brainin P, Hoffmann S, Fritz-Hansen T, Olsen FJ, Jensen JS, BieringSørensen T. Usefulness of Postsystolic Shortening to Diagnose Coronary Artery Disease and Predict Future Cardiovascular Events in Stable Angina Pectoris. J Am Soc Echocardiogr 2018; 31: 870-9. [Crossref]

18. Onishi T, Uematsu $M$, Watanabe $T$, Fujita $M$, Awata $M$, lida 0 , et al. Objective interpretation of dobutamine stress echocardiography by diastolic dyssynchrony imaging: a practical approach. J Am Soc Echocardiogr 2010; 23: 1103-8. [Crossref] 